\title{
High Quality Sensor Placement for SHM Systems: Refocusing on Application Demands
}

\author{
Bo Li \\ Dept. of Civil and Structural Eng. \\ The Hong Kong Polytechnic Univ. \\ cejoe@polyu.edu.hk
}

\author{
Dan Wang*, Feng Wang \\ Dept. of Computing \\ The Hong Kong Polytechnic Univ. \\ \{csdwang, csfwang\}@ comp.polyu.edu.hk
}

\author{
Yi Qing $\mathrm{Ni}^{\dagger}$ \\ Dept. of Civil and Structural Eng. \\ The Hong Kong Polytechnic Univ. \\ ceyqni@ polyu.edu.hk
}

\begin{abstract}
There are heavy studies recently on applying wireless sensor networks for structural health monitoring. These works usually focus on the computer science aspect, and the considerations include energy consumption, network connectivity, etc. It is commonly believed that for the current resource limited wireless sensors, system design could be more efficient if the application requirements are incorporated. Nevertheless, we often find that, rather than integration, assumptions have to be made due to lack of knowledge of civil engineering; for example, to evaluate routing algorithms, the sensor placement is assumed to be random or on grids/trees. These may not be practically meaningful to the respective application demands, and make the great efforts by the computer science community on developing efficient methods from the sensor network aspect less useful.

In this paper, we study the very first problem of the SHM systems: the sensor placement and focus on the civil requirements. We first study the current general framework of structure health monitoring. We redevelop the framework that includes a new sensor placement module. This module implements the most widely accepted sensor placement scheme from civil engineering but focusing on its usefulness for computer science. It provides such interfaces that can rank the placement quality of the candidate locations in a step by step manner. We then optimize system performance by considering network connectivity and data routing issues; with the objective on energy efficiency.

We evaluate our scheme using the data from the structural health monitoring system on the Ting Kau Bridge, Hong Kong. We show that a uniform and a state-of-the-art placement are not very meaningful in placement quality. Our scheme achieves almost the same sensor placement quality with that of the civil engineering with five-fold improvement in system lifetime. We conduct an experiment on the in-built Guangzhou New TV Tower, China; and the results validate the effectiveness of our scheme.
\end{abstract}

\section{INTRODUCTION}

Sensor networks today are widely used for structural health monitoring (SHM). Examples include the monitoring systems of the Guangzhou New TV Tower, China (GNTVT [1], See Fig. 16 (a)) and the Ting Kau Bridge, Hong Kong [16], to name but a few. In the computer science community, it is well accepted that the resource optimization of the sensor systems must be tightly correlated with the respective applications [6]. This is sharply different from the Internet, where the communication system is strictly separated from the above applications and only sees general packets. As such, schemes like innetwork data aggregation [7], data-aware sensor and relay

\footnotetext{
*Dan Wang's work is supported by grant PolyU/G-YG78A-PB0R, A-PJ19, 1-ZV5W, and RGC/GRF PolyU 5305/08E.

†Yi Qing Ni's work is partially supported by a grant from The Hong Kong Polytechnic University through the Development of Niche Areas Programme (Project No. 1-BB68).
}

node placement [23] etc, are developed. Nevertheless, most data aggregation studies focus on statistics such as MEDIAN, AVERAGE, SUM and VARIANCE. And the sensor placement is commonly assumed to be random or on specific grids/trees. These may not be practically meaningful for the respective applications; making the great efforts in the computer science community less useful.

For such cross-discipline applications, on one hand, both the civil engineering and computer science should focus on and contribute their own expertise. It is unwise that one community crosses the line into the study of the other too much. On the other hand, there is no one line boundary of the responsibilities, and there exists overlapping area. That is, it may not be appropriate to assign some design issues specifically to civil engineering or to computer science.

A question we faced was who should undertake the study of such overlapping area. Our experience shows that the civil engineering is usually less motivated. After all, their objective is the health of the structures, which, they have their own difficulties to achieve. They are less willing to study how to compromise the quality of structural evaluation to improve the efficiency of the sensor systems. They are, however, happy to make choices if reasonable options are given.

In this paper, we focus on the very first problem in the SHM applications: where the sensors to be placed, that can best capture the structure properties and is suitable for efficient system design. In previous studies, one common assumption in the computer science community is that the sensor placement should be determined by civil engineering. The computer science then designs the sensor networks with these locations fixed. As said, it is not true that the civil engineering has a hard line on the sensor placement. It is just whether the locations suggested are reasonable for them.

Looking into the sensor placement schemes from the computer science community, the sensors are either randomly deployed or in some regular forms such as grids or trees. The data collected using such deployments are not entirely meaningful. We illustrate this with the following example.

An Example: We consider a 2-D wall structure connected to the ground with seven joint points, see Fig. 1. Assume that there are 49 candidate locations $(7 \times 7)$. Assume we have a limited number of sensors and have to select a subset of locations from the candidate locations. In civil engineering, there is a well accepted metric to specify the placement quality, the Fisher Information Matrix (FIM) determinant (to 
TABLE I

ABBREVIATION DESCRIPTION

\begin{tabular}{c|l}
\hline Abbreviation & Full Name \\
\hline FIM & Fisher Information Matrix (placement quality indicator) \\
EFI & EFfective Independence placement method \\
FEM & Finite Element Model
\end{tabular}

be explained later, for abbreviation description please refer to Table I); and the larger the FIM is, the better the quality. We compare the FIM between a civil oriented placement method $(\mathrm{EFI})$ and the uniform method (constructing an $n \times n$ grid), which could be a likely choice by computer scientists due to lack of knowledge of civil engineering. The result is shown in Table II, and the placement of 16 sensors of the two methods is shown in Fig. 1. Clearly the fewer sensors deployed, the less the FIM determinant. We thus assume that a placement on all 49 locations to have a 100\% FIM and normalize the FIM determinant of all other placements. While it is not surprising that EFI performs better, the difference between EFI and the uniform is quite substantial. For example, when deploying 36 sensors $(6 \times 6$ grid), the FIM of the uniform placement is roughly half of that of the EFI and the FIMs of 25 and 16 sensors are only one third of that of the EFI. If we interpret this in another way, by deploying 16 sensors with the EFI method, the performance (in terms of FIM) is almost the same as uniform placement with 25 sensors. In other word, we can save more than $50 \%$ of sensors just by deploying the sensors on better locations.

In this paper, we first provide an understanding of the current SHM mechanism, where the monitoring is divided into in-construction monitoring and in-service monitoring. We argue that the current wireless mote-like sensor can play an important role in the in-construction monitoring. We reorganize the SHM mechanism and develop a new sensor deployment module which provides open interfaces for the computer science optimization. We release this module as an open source package. One important function of this module is that given the set of candidate locations, the module sorts these locations according to their contributions to the FIM determinant. In other word, it can provide the location quality in a step by step manner. Consequently, rather than having a fixed and completed sensor placement, computer scientists can optimize iteratively with different sensor placement.

We then jointly design the sensor placement with energy efficiency, which is an important metric for the system performance in computer science. We also consider such factors as the network connectivity and data routing. An optimization problem is formulated. As the search space for an optimal solution is large, we develop an efficient local search subroutine.

We evaluate our scheme with a comprehensive set of evaluation. We apply the model and data from a real structure Ting Kau Bridge in Hong Kong. This system, though using wired sensors, involves more than 230 sensors and accomplishes 24-hour monitoring. We study various state-of-the-art power aware routing algorithms and relay node placement algorithms developed by computer science. We do see that these algorithms significantly increase the system lifetime against straight forward deployment. Yet, the data they collected are

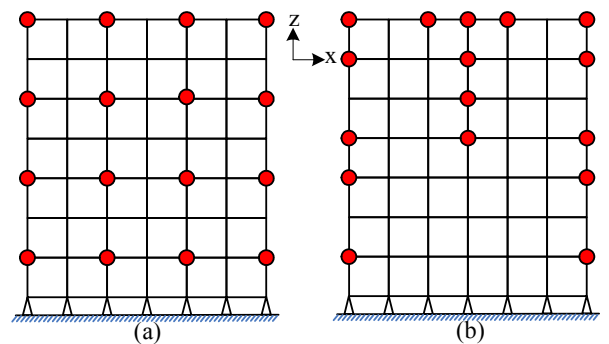

Fig. 1. A 2-D wall model (a) Uniform placement. (b) Placement with EFI.

TABLE II

FIM DETERMINANT $|Q|$

\begin{tabular}{|c|c|c|}
\hline Sensor No. & EFI & Uniform \\
\hline 49 & $100 \%$ & $100 \%$ \\
\hline 36 & $59 \%$ & $35 \%$ \\
\hline 25 & $24 \%$ & $8 \%$ \\
\hline 16 & $7 \%$ & $2 \%$ \\
\hline
\end{tabular}

far from satisfactory. We show that our algorithm can achieve almost the same FIM as compared to the scheme used in civil engineering, with a 2 to 5 folds improvement in system lifetime, which is comparable to the algorithms developed in computer science. We also conduct real experiments on the in-built Guangzhou New TV Tower, to be completed at the end of 2009, and we obtain similar results.

As a summary, the contributions of this paper are 1) We are the first to study a problem in an overlapping area for the SHM systems, the sensor placement. This task is by no means easy as it involves inter-discipline knowledge. We show, however, the effort is rewarding. 2) We develop a new sensor placement module which incorporates (and hides) the civil requirements and provides interfaces that make further design in computer science easier and more flexible; 3) We show a sensor network design using the new module which focus on computer science system efficiency. 4) We evaluate our algorithms with the data from a real SHM system on the Ting Kau bridge; and real experiments on the Guangzhou New TV Tower, which is the first SHM system on a high-rising tower structure.

The remaining part of the paper is organized as follows. In Section II, we present related work. Section III describes the general SHM framework, our architecture design and the new sensor placement module which evaluates the sensor placement quality and provides useful interfaces for computer science optimization. We discuss the problem and the detailed algorithms in Section IV and Section V, respectively. Section VI includes a comprehensive evaluation using the data from the Ting Kau Bridge. Our experimentation with real sensors on the Guangzhou New TV Tower is shown in Section VII. Finally, section VIII concludes the paper.

\section{RELATED WORK}

In the past years, sensor networks have been experimented in various applications, such as habitat monitoring [14], volcano monitoring [22], etc; most of which are cross-discipline. The capability and limitation of the current wireless sensor networks have been heavily studied. The sensors (not necessarily the mote-like sensors) are expected to be resource limited so that they can become cheap and small. Consequently, application requirements are to be taken into serious 
consideration so as to jointly optimize the system performance. This may require an expertise of non-computer science related field. By no means such a task is easy. The benefit may be rewarding, however. An interesting recent example is the study of the sea flow [4], which introduced a meandering water movement model from oceanography. This fosters follow-up efficient designs in sensor networks [13].

In this paper, we study sensor networks for structural health monitoring. One comprehensive overview on applying wireless sensors into SHM can be found in [21]. SHM is an emerging field and more and more structures have integrated real SHM systems, e.g., the SHM in Ting Kau Bridge [16], etc. Wired sensors are still dominated in these SHM applications. It is gradually accepted, however, that wireless sensor systems have some intrinsic advantages; and for the SHM that we are working on for the Guangzhou New TV Tower [17], wireless system is partially adopted.

There have been existing studies using the state-of-theart wireless sensor networks for the SHM applications; and testbed experiments have been carried out [9][24]. These systems have validated from the computer science aspect that the current wireless sensor networks can meet the sampling rate, data throughput of the SHM applications. One limitation is still the system working time. In all these studies, the civil engineering requirement is only marginally considered.

There is a recent framework [5] that effectively integrates a distributed damage detection algorithm (DLAC). By novelly trading off the computational capacity with communication transmission, a system lifetime of over 190 days is achieved using Imote-2 sensors. Our work on sensor placement is orthogonal to this study. One reason that we start with the sensor placement rather than the damage detection module is that though a number of damage diagnosis methods have been proposed, feasibility of these methods for real-world applications, especially for large-scale structures has been rarely examined, a gap still exists between research and practice [17]. On the other hand, the FEM model and sensor placement using the EFI method that we study in this paper and incorporated in our software package have become a benchmark standard in civil engineering and thus universally applicable.

There is a near optimal sensor placement algorithm where the sensor placement is chosen to be informative and energy efficient [10]. Entropy is used to measure the quality of the data collected and the placement is gradually refined by a nonparametric Gaussian Process. The meaning of informative in [10] is thus very general. Our sensor placement is specific for the SHM applications and the derivation of the FIM and EFI all have their physical meanings. We believe the algorithm in [10] can be used in diverse applications beyond SHM of general sense, but may not be suitable for specific SHM applications.

\section{ARChitecture Design}

\section{A. Background of the SHM Framework and Sensor Placement}

1) The General SHM Framework: The general routine for structural health monitoring is usually divided into two phases: the in-construction monitoring and the in-service monitoring.

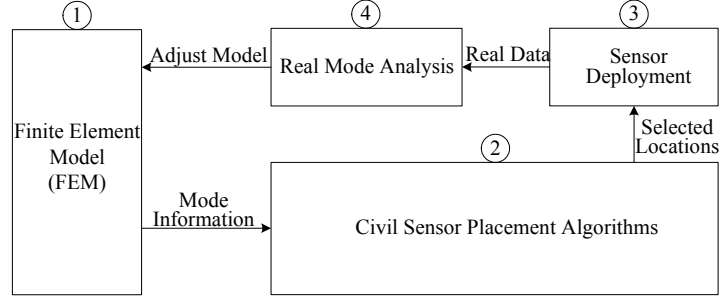

Fig. 2. A general SHM framework.

In the first phase, the objective is to simultaneously establish and calibrate the Finite Element Model (FEM) [17] of the structure. The FEM model contains the information of the entire property of the structure. This model will be used for validation of the in-service monitoring as well as large-scale damage detection during the in-service monitoring. The second phase starts after the structure is completed and the FEM model is built. The sensors periodically collected the data to validate whether the data conform to the FEM model.

In these two phases, the sensors used could be different. The second phase (in-service monitoring) can be a very long time (tens of years) and thus wire-power-equipped sensors are still the best choice under the current technology. In the first phase, wireless sensor networks are highly welcomed as the wires are so easily damaged during structure construction and this causes the notorious head-ache for the SHM systems deployment. ${ }^{1}$ The life-span of the sensor networks in this phase is usually in the order of months longer. We believe that a careful management of the sensor network is possible to achieve such lifetime span.

In the in-construction phase, an iterative procedure is used to calibrate the FEM (See Fig. 2). The initial FEM usually is the FEM of a similar or simpler structure. The mode information will be an input of any sensor placement algorithm of civil engineering and the output will be a placement scheme. The sensor will be deployed and collect data for a period of time (e.g., two months). After the construction of the structure progresses, these data will be used to calibrated for a more refined FEM; and a new iteration starts.

2) Sensor Placement: The sensor placement is a key module for building FEM. There are many sensor placement algorithms in civil engineering and EFI is the most widely accepted. The sensor placement quality is reflected by FIM determinant. The larger the FIM determinant, the better the placement. In EFI, given a set of candidate locations, a set of mode shapes (intuitively, one may regard it as what a structure roughly look like), and the number of sensors, EFI can output the best set of locations for the sensor placement. To smooth the exposition, we delay a more detailed discussion of the EFI algorithm in the Appendix.

\section{B. The New Framework and SPEM}

The SHM systems developed by civil engineering do not consider the constraints of the wireless systems from computer

\footnotetext{
${ }^{1}$ During hammers and drills of structure construction, it is unavoidable that the wires of the SHM systems are damaged. Usually the structure and the SHM system are developed by different companies. This makes the staffs for structure construction even less responsible to protect the SHM systems.
} 


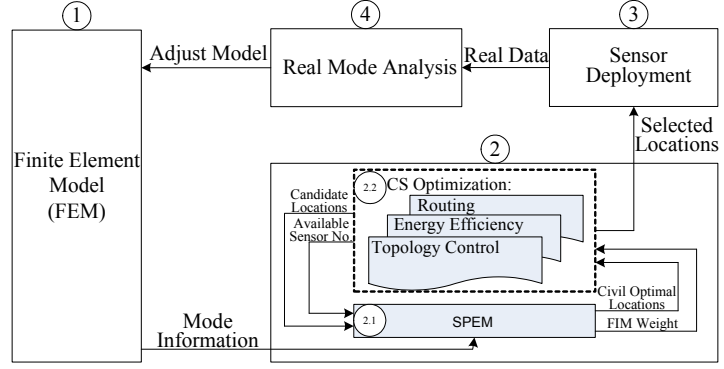

Fig. 3. The New SHM framework.

science. We reorganized that the architecture as shown in Fig. 3. We insert the common constraints of computer science into the sensor placement; where topology control, traffic aware routing and energy efficiency can be jointly considered.

We design a special module Sensor Placement using EFI method (SPEM), which works as a transition module between the FEM and the sensor placement algorithms. SPEM is implemented by MATLAB, which is widely supported in civil engineering [3]. Currently it provides interfaces that can not only be accessed by MATLAB, but also by $\mathrm{C}$ and $\mathrm{C}++$ programs, which are more suitable for the computer science community. SPEM monitors the data quality of the placement. It provides useful information for computer science and hides the civil details. We release SPEM as an open source package at [11] and a demo of SPEM can be found in [12].

The core algorithm of the package is based on the EFI method [8]. The key of SPEM is that it provides a step by step selection. This is important as with the interface of SPEM, the resource optimization of the computer science constraints can be iteratively and interactively adjusted with the civil placement quality. A joint consideration is thus possible.

More specifically, the inputs of SPEM include 1) mode shape information of a structure (obtained from FEM module); 2) the set of candidate sensor locations; and 3) the number of sensor available. As shown in Fig. 3, the CS Optimization module can call SPEM as a sub-function with input 2) and 3). The outputs of SPEM include 1) the selected Civil optimal sensor locations, and 2) the FIM weights for each location. The CS Optimization module can then re-consider with computer science constraints. After iterative adjustment, the final placement is output and the data collection starts.

For the CS Optimization module, different applications can incorporate different factors. In this paper, we consider the following three, 1) topology control, where all the sensor nodes must be connected, 2) data routing, the data routing in sensor network has a many to one pattern; and 3) energy efficiency. For wireless sensor networks, energy consumption is dominated by communication. The traffic volume and communication range play the two most important factors. We detail the design in the rest of the paper.

\section{The Sensor Placement Problem in SHM}

\section{A. Preliminaries}

Assume the structure has $M$ feasible locations for sensor deployment, with the base station placed at location $s_{0}$. The $M$ can be arbitrary large if we refine the granularity of the resolution of the surface. The number of sensors are limited. Assume there are $N(<M)$ sensors to be attached by some location assignment $S=\left\{s_{1}, s_{2}, \ldots, s_{N}\right\}$ where sensor $i$ is placed at location $s_{i}$. Let $Q$ and $|Q|$ denote the FIM and its determinant given by a location assignment.

Let the maximum communication range of a sensor be $R_{\max }$. We assume that the communication range of each sensor can be dynamically adjusted. Given a routing algorithm, let the path from a source sensor to the base station be $p=x_{0} x_{1} \ldots x_{k}$. Define $p[i]=x_{i}$ as the $i$-th hop sensor on path $p$ and $\gamma_{p}$ as the amount of traffic that travels along path $p$ within one round of data collection. Let $E_{r e c v} / E_{\text {send }}$ represent receiving/sending energy consumption. The energy consumption of sensor $i$ can then be computed as:

$$
\begin{aligned}
E_{i}= & \sum_{\forall p, \exists j>0, p[j]=s_{i}} \gamma_{p} \cdot E_{\text {recv }}(\overline{p[j] p[j+1]}) \\
& +\sum_{\forall p, \exists j, p[j]=s_{i}} \gamma_{p} \cdot E_{\text {send }}(\overline{p[j] p[j+1]}),
\end{aligned}
$$

where $\overline{p[j] p[j+1]}$ denotes the distance between the two sensors at locations $p[j]$ and $p[j+1]$. Let $E=\max _{i=1 . . N} E_{i}$, which is the maximum energy cost on one sensor. We define the system lifetime $T$ to be the total rounds of data collection before any battery runs out of energy. This can be calculated by $T=\mathbb{E} / E$, where $\mathbb{E}$ is the energy reserve on one sensor.

\section{B. Problem Statement}

We formulate the sensor placement problem in SHM as finding a location assignment $S=\left\{s_{1}, s_{2}, \ldots, s_{N}\right\}$ out of $M$ possible locations for the $N$ given sensors subjecting to the following constraints:

(1) Data Delivery Constraint:

$$
\forall p=x_{0} \ldots x_{k} \text { used for data delivery, }
$$

$$
\overline{p[j-1] p[j]} \leq R_{\max }, j=1 \ldots k ;
$$

(2) Connectivity Constraint:

$$
\begin{gathered}
\forall i=1,2, \ldots, N, \exists p=x_{0} \ldots x_{k}, \\
p[0]=s_{i}, p[k]=s_{0} .
\end{gathered}
$$

Our objective is to maximize both $|Q|$ and $T$, i.e., the FIM (sensor placement quality) and the system lifetime. Since $\mathbb{E}$ is a given constant, $T$ is solely depending on $E$. We use a general objective function of $f(|Q|, E)$, which can be written into different forms according to different emphasis on the FIM quality and the system lifetime. In this paper, we will study $f(|Q|, E)=\frac{|Q|}{E}$. The physical meaning can be interpreted as the FIM quality gain by per unit energy. The objective is to maximize $f(|Q|, E)=\frac{|Q|}{E}$.

\section{Algorithm Design}

Our problem is affected by many factors. Even the location assignment $S$ is given, the energy consumption is still affected by the data routing scheme. Instead of a joint optimization (the problem will be an NP-hard problem even only the data routing and topology control are considered), we choose to decouple the data routing as a separate module. 


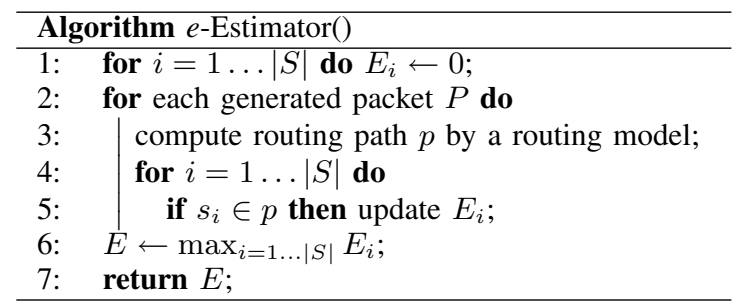

Fig. 4. The $e$-Estimator algorithm.

In this section, we first develop an open sub-routine $e$ Estimator() that can easily estimate $E$ from any routing protocol. We then introduce our solution which, by exploiting our software package SPEM, can greatly reduce the searching space while providing high quality location assignments.

\section{A. Energy Consumption Estimation}

The maximum energy consumption of a candidate location assignment depends on the routing protocol used by the data collection application. This falls into the domain of power aware routing. Five general power-aware routing models were proposed in [19], and some other results can be found in [2][20]. The intuition behind is usually to route packets through the nodes that have higher residual power.

In SHM applications, each sensor periodically generates roughly the same amount of data in each round. As such, the energy consumption on each sensor can be estimated apriori. The study of different routing protocols is out of the scope of this paper; for illustration purpose, we use the Shortest Path routing model and Per Packet Cost Minimization model for our $e$-Estimator() module. We summarize the two models as follows and a detailed description can be found in [19]:

The Shortest Path Model (SP): In this model, a shortest path tree is built rooted at the base station. We use the Euclidian distance as the metric for the shortest path model.

The Per Packet Cost Minimization Model (PPCM): In this model, each packet is sent on a path that minimizes the total energy consumption to deliver this packet. Specifically, let $g\left(s_{i}\right)$ denote the energy consumption of sensor $i$ for delivering this packet. Let the path a packet $P$ travels be $p=x_{0} \ldots x_{k}$, the total energy consumption is

$$
C_{P}=\sum_{j=0}^{k} g\left(x_{j}\right) .
$$

Our $e$-Estimator algorithm is shown in Fig. 4. The input is a location assignment $S$; and different routing models can be applied in line 3. It is worth noting that for some routing models (e.g. PPCM), routing packets generated by different order can lead to different energy consumption. In such case, we run $e$-Estimator() with different orders and take the average as the estimated maximum energy consumption.

\section{B. High Quality Location Assignment}

The solution space of an optimal location assignment $S$ has the complexity of $O\left(N^{M}\right)$ and $M$ can be very large. In this subsection, we study an algorithm with local search that is simple for implementation and efficient.

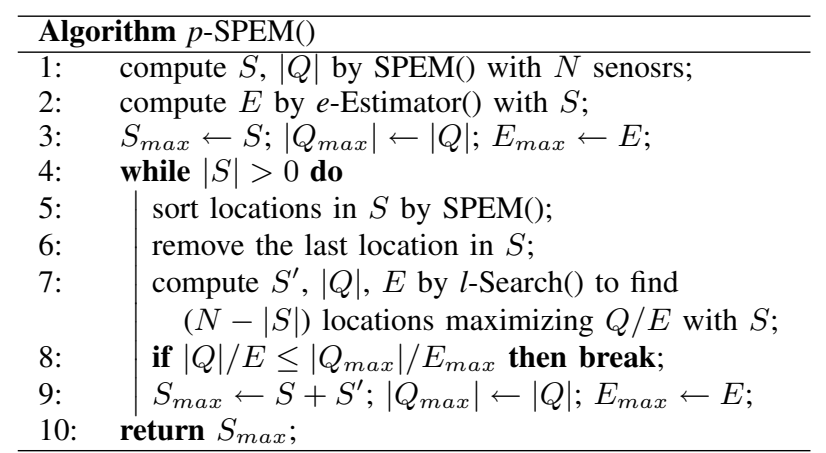

Fig. 5. The $p$-SPEM algorithm.

We propose an algorithm named Power-aware SPEM, shortened as $p$-SPEM, see Fig. 5. The algorithm first call SPEM with $M$ candidate locations for sensor placement and the number of sensors $N$ as input. The output $N$ locations for sensor placement is best suitable for civil requirement (line 1). We then adjust the $N$ locations to better fit both the computer science and civil constraint. The idea is to improve iteratively the system lifetime by the sensors with the least contribution to the FIM quality. Specifically, in each iteration (line 4-9), we remove the sensor at the location that contributes least to FIM from $S$ and put it together with other previously removed sensors to some other locations $S^{\prime}$ so as to improve the system lifetime and maximize $|Q| / E$. The iteration proceeds until no further improvement $|Q| / E$ can be observed. We will show in our simulation that this procedure converges quickly.

To search for the suitable locations $S^{\prime}$, we further design a local search algorithm $l$-Search(). Note that if we do not perform $l$-Search(), the algorithm is greedy in terms of $|Q| / E$. The algorithm takes $M$ feasible locations, the number of given sensors $N$, the location assignment after sensor removals $S$ and a search distance parameter $d$ as input and finds locations for the removed sensors to maximizes $|Q| / E$. The algorithm first places these removed sensors one by one in a greedy manner, where each placed sensor maximizes $|Q| / E$ when being placed. Then a local adjustment is performed iteratively. In each iteration, all sensors try the neighbor locations that are within a distance $d$; the neighbor location that maximally improves $|Q| / E$ will be selected and the corresponding sensor is then adjusted to that location. The iteration stops when no improvement can be found. We also randomly select the start locations so as to avoid stucking at local optimums. We summarize the algorithm in Fig. 6.

Comparing with the exhaustive search, our solution reduces the complexity from $O\left(N^{M}\right)$ to $O\left(\sum_{i=1}^{N}(i \cdot M+i \cdot M \cdot n \cdot i)\right)$, where $n$ is the maximum number of neighbor locations. This complexity is bounded by $O\left(N^{4} M\right)$.

\section{Vi. Performance Evaluation}

\section{A. Metrics and Methodology}

We evaluate the performance of our algorithms through an identified bridge model based on a real structure, Ting Kau Bridge in Hong Kong (Fig. 7), which is a $1,177 \mathrm{~m}$ long multiscan cable-stayed bridge with three towers supporting two main spans of $448 \mathrm{~m}$ and $475 \mathrm{~m}$ respectively and two side spans 

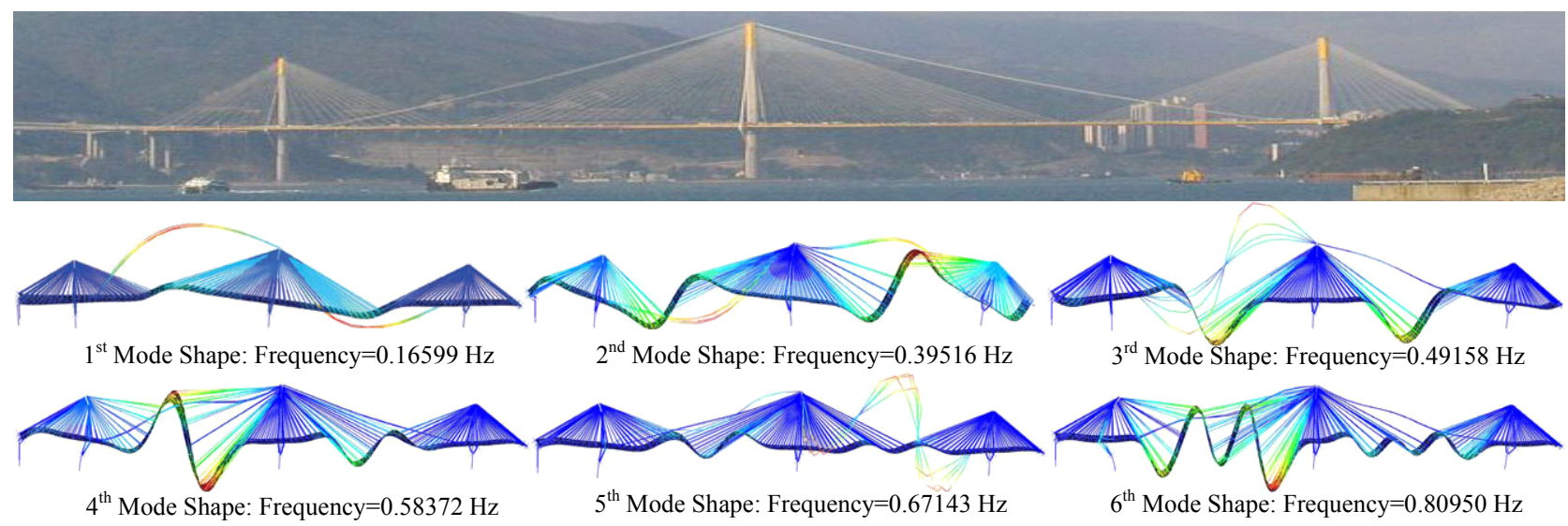

Fig. 7. Bird view of Ting Kau Bridge and six identified global mode shapes.

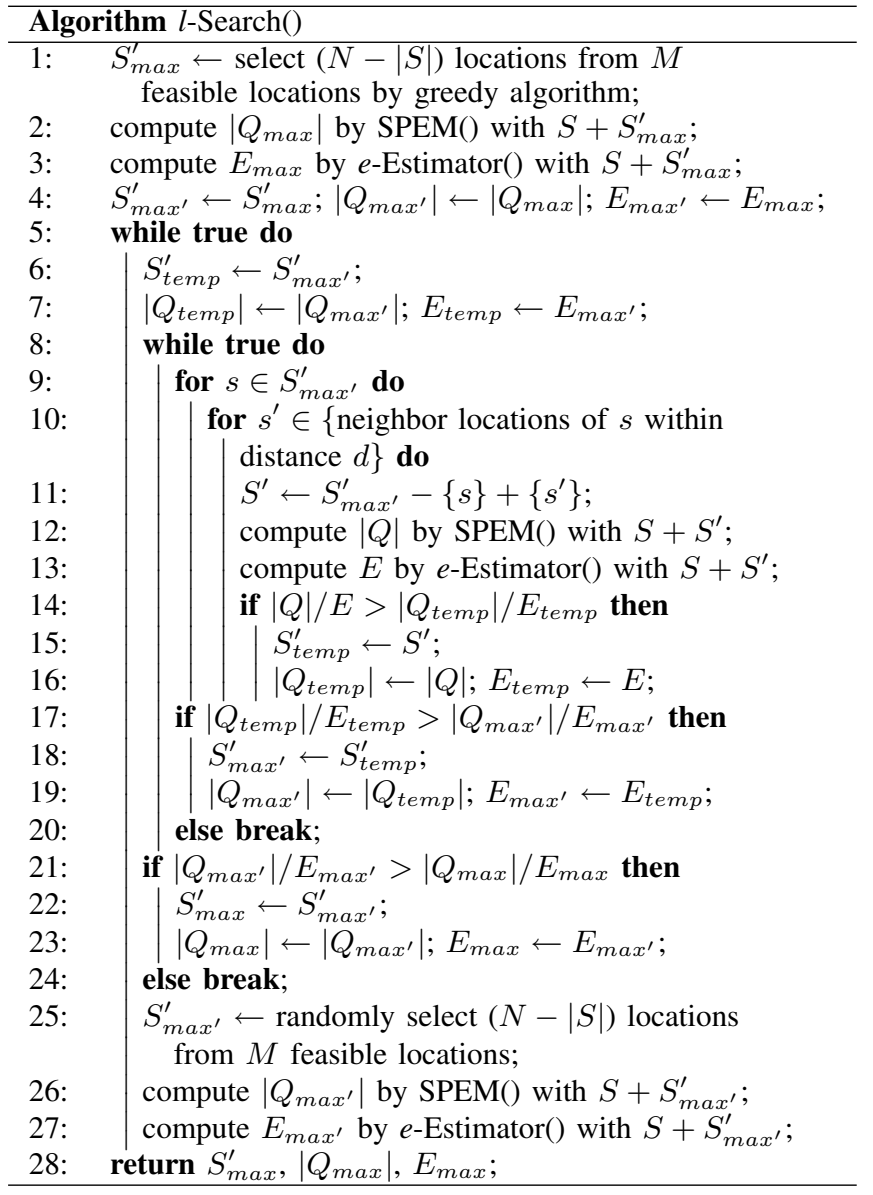

Fig. 6. The $l$-Search algorithm.

of $127 \mathrm{~m}$ each. After its completion in 1998, the bridge was instrumented with a long-term structural health monitoring system by the Highways Department of the Hong Kong SAR Government for a 24-hour monitoring. A precise threedimension (3D) FEM model of the Ting Kau Bridge, calibrated using real data collected by its lifelong SHM system, has been developed with a global mode precision higher than $93 \%$ in [15]. In this model, all geometric and material properties are accurately represented without further approximation. In our experiments we recover the first 6 mode shapes (see Fig. 7) with totally 272 feasible locations for sensor placement.
To compute the maximum energy consumption $E$ and the system lifetime $T$, we use the following well-accepted energy model for packet transmission [18] in our evaluation:

$$
E_{\text {send }}(r)=a r^{\alpha}+b \text {, }
$$

which can also be normalized as

$$
E_{\text {send }}(r)=r^{\alpha}+c,
$$

here $r$ is the communication range, $\alpha$ is an exponent parameter in $[2,6]$ and $c$ is a small constant comparing with $r^{\alpha}$. The energy consumption for packet receiving is given by

$$
E_{\text {recv }}(r)=c \text {. }
$$

We set the parameters to be $\alpha=2$ and $c=4500$ [18].

For comparison, we implement another three schemes, namely, the EFI method, Pure Uniform solution and Uniform solution for Data Collection. The EFI method is exactly the one described in Section III, which provides the best location assignment from the civil engineering aspect. The Pure Uniform solution deploys the sensors by the uniform distribution, which could be a choice by the some developers. The Uniform solution for Data Collection is a state-of-the-art approach proposed by computer scientists [23], designed specifically to extend system lifetime for sensor data collection. The main idea is to divide sensors into two groups, where one group is uniformly deployed to collect sensing data while the other group is deployed by some computed density function to relay traffic from the sensors in the first group to the base station.

We will use the FIM quality and system lifetime (measured by the first depleted sensor) as the two main metrics to evaluate the performance of our algorithms.

\section{B. Simulation Results}

We first evaluate the impact of the number of sensors. From Fig. 8 and Fig. 9, we can see, as expected, the FIM quality increases with the number of used sensors for all strategies. However, our solution performs much better (about 2 to 5 orders of magnitude) than the two uniform approaches and stays very close to the EFI method, which is known to be the best solution in term of the FIM quality. The two uniform approaches and the PPCM model do not show much difference in the FIM quality and are far from satisfactory. Their poor performance is because their deployments are 


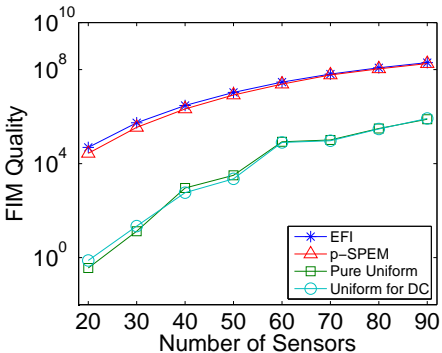

Fig. 8. FIM quality of different sensor amount under SP model.

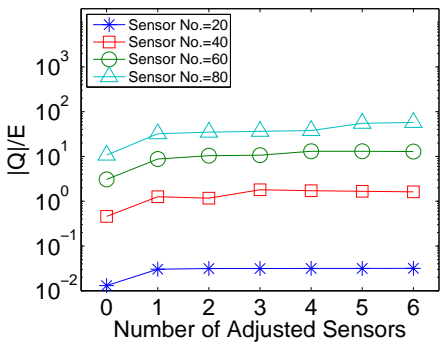

Fig. 12. $\frac{|Q|}{E}$ as a function of adjusted sensor number under SP model.

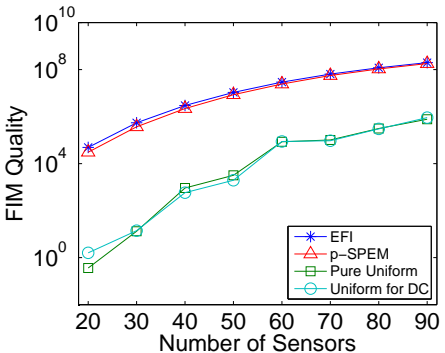

Fig. 9. FIM quality of different sensor amount under PPCM model.

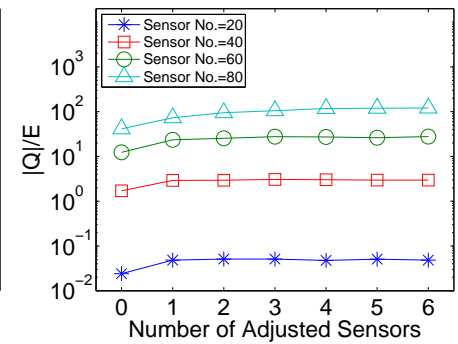

Fig. 13. $\frac{|Q|}{E}$ as a function of adjusted sensor number under PPCM model.

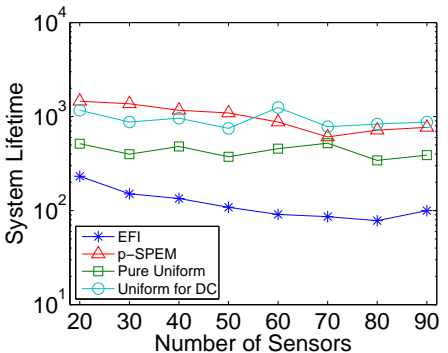

Fig. 10. System lifetime of different sensor amount under SP model.

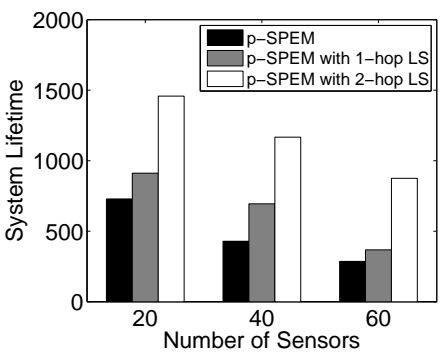

Fig. 14. System lifetime with diverse searching ranges under SP model.

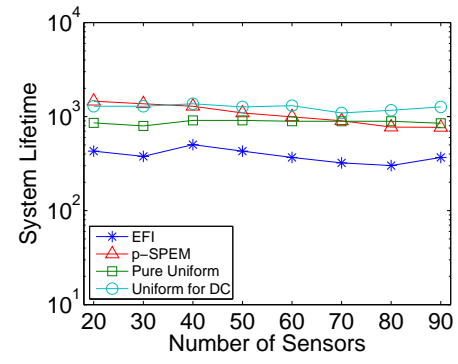

Fig. 11. System lifetime of different sensor amount under PPCM model.

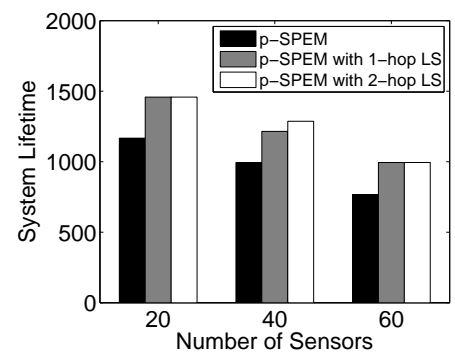

Fig. 15. System lifetime with diverse searching ranges under PPCM model. oblivious to the application demand from SHM. This has clearly justified the main theme of our effort: for the SHM applications using sensor networks, a study on efficiently and jointly consideration of both the civil and computer science aspects is as important as the studies focusing on each single discipline. We conjecture that this could be true for most sensor network applications today; this is in sharp contrast to the Internet study. Another observation that worth mention is that when the number of sensors is larger, the gap of the FIM quality between different methods is smaller. This is because the total candidate locations is always 272 and the FIM for all methods will become similar when there are more and more sensors deployed.

We next examine the system lifetime and the results are shown in Fig. 10 and Fig. 11. We can see that our solution is close to the two uniform approaches and is roughly 2 to 5 times (note that the $y$-axis is in log-scale) of the EFI approach. Also, an interesting observation is that the number of senors does not affect the system lifetime much. A closer look reveals that the more sensors, the more data (no aggregation). Since the placement is on a bridge, which is not a square or circle-shaped sensing field, the data traffic may not be evenly distributed by simply adding more sensor nodes.

Comparing the results by the two different routing models, the SP model and the PPCM model, it is clear to see that the FIM quality is not sensitive to the used routing model. This is because that the FIM quality only depends on the locations where sensors are placed. On the other hand, the impacts of routing models on the system lifetime are more noticeable, where the system lifetime achieved by PPCM is up to 4 times of that achieved by SP. An intuitive explanation is that PPCM adopts the power-aware principle and dynamically routes packets along paths with high residual energy on each sensor (recall the model description in Section V). This shows the effort achieved by the computer science community is noticeable. This can also be seen comparing Uniform for DC and Pure Uniform. The former performs much better in system lifetime. Yet they all suffer from poor FIM quality. On the other hand, in spite of the different routing models, our solution uniquely provides high quality sensing data and greatly extends system lifetime.

We next study some internal parameters of our algorithm. We show in Fig. 12 and Fig. 13 the number of adjusted sensors (line 4-9 in $p$-SPEM). We see that the algorithm converges very fast. After adjusting 3 to 4 sensors, our algorithm is already stable. In addition, this is very little affected by the total number sensors to be deployed. This shows that our algorithm is of very low complexity in practice.

We next show the performance of our $l$-search subroutine. From Fig. 14 and Fig. 15, the system lifetime does increase with searching a small range of neighbor locations and thus our $l$-search subroutine is useful. Note that $i$-hop $\operatorname{LS}(i=1,2)$ means Local Search(LS) is within $i$ hop.

\section{EXPERIMENTS ON GNTVT}

We conduct a field experiment to collect real mode shapes of the Guangzhou New TV Tower. We would like to mention that though deployments of SHM systems on bridges have been widely reported (as the Ting Kau Bridge), SHM systems for high-rising structures are very rare [17]. The GNTVT, to be completed by the end of 2009 , will become the tallest TV tower in the world with a height of $610 \mathrm{~m}$, with a $156 \mathrm{~m}$ antenna (see Fig. 16 (a)). Our experiment measurement, conducted on January 2, 2009, was the first measurement of GNTVT when the main tower reached to a full height of $454 \mathrm{~m}$.

\section{A. System Deployment}

The experiments herein not merely aim to validate the effectiveness of the proposed $p$-SPEM algorithm, but also 


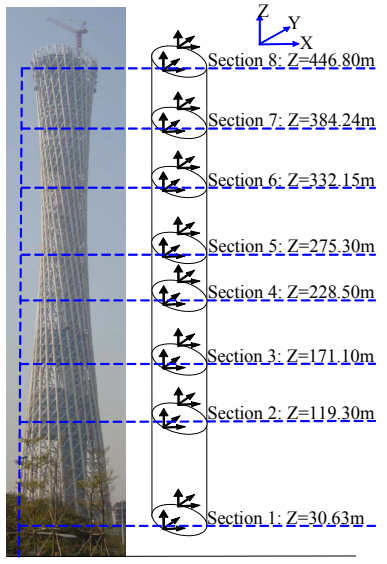

(a)

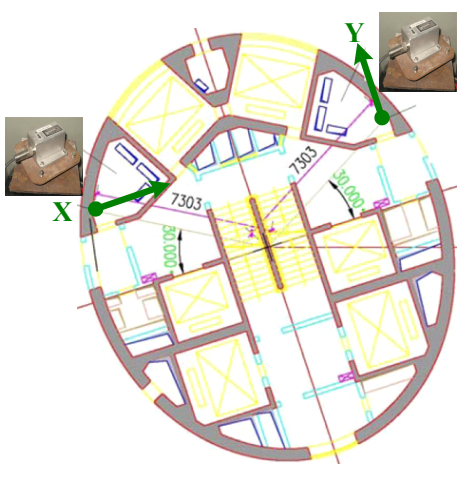

(b)
Fig. 16. Sensor deployment on GNTVT during the in-construction phase. (a) The GNTVT tower (currently 550m) and the 8 set of locations for the sensors. (b) Sensor deployment on each floor (two sensors).

emphasized on the framework in Fig. 3. In our experiment, we first adopt a raw FEM model of GNTVT to generate mode shapes. We use the EFI method to select 11 sensor locations as a candidate set $(5.20 \mathrm{~m}, 30.63 \mathrm{~m}, 77.10 \mathrm{~m}$, $119.30 \mathrm{~m}, 171.10 \mathrm{~m}, 228.50 \mathrm{~m}, 275.30 \mathrm{~m}, 288.10 \mathrm{~m}, 332.15 \mathrm{~m}$, $384.24 \mathrm{~m}$ and $446.80 \mathrm{~m})$. After considering the difficulties of field deployment (e.g., the feasibility of some floors of GNTVT), we choose 8 locations and a total of 8 sensor sets are deployed onto these floors $(30.63 \mathrm{~m}, 119.30 \mathrm{~m}, 171.10 \mathrm{~m}$, $228.50 \mathrm{~m}, 275.30 \mathrm{~m}, 332.15 \mathrm{~m}, 384.24 \mathrm{~m}$ and $446.80 \mathrm{~m})$.

On each floor we deployed two sensors, one for the $X$ direction and the other for the $Y$-direction. We adopt high precision accelerometers Tokyo Sokushin AS-2000 for acceleration monitoring. Though the deployed accelerometer is able to acquire vibration status of the tower, we found the sensed vibration amplitude is very small. To increase the signal strength and the SNR, a signal amplification and conditioning board is developed using TI PGA202. This board works as an amplification middle-ware between the accelerometer and analogue input of our data collection motes. Fig. 16 illustrates the deployment details.

After the sensor deployment (step 3 of Fig. 3) on GNTVT, a continuous data collection is conducted to collect acceleration data for 24 hours. Using the collected real data, mode information GNTVT is analyzed and the FEM of GNTVT is calibrated (step 4 of Fig. 3). After the calibration, the FEM of GNTVT becomes more precise. Using this new FEM, a new set of three mode shapes of GNTVT are recovered $(0.1623 \mathrm{~Hz}, 0.5216 \mathrm{~Hz}$ and $1.0288 \mathrm{~Hz}$ respectively). These mode shapes are used in step 2 of Fig. 3, where $p$-SPEM is applied to take a joint optimization of both civil and computer science constraints. We summarize the placement results as follows.

\section{B. Experiment Results}

Fig. 17 and Fig. 18 summarize the results on FIM Quality and system lifetime. As expected, in all the three placement methods, the FIM Quality in Fig. 17 increase as the number of sensors increases. The EFI outperforms $p$-SPEM and the Uniform method. $p$-SPEM ranks the second and the performance is closer to the EFI method. In Fig. 18, the system lifetime of $p$-SPEM are much better than EFI in all results except for the 9-sensor case. Looking into the details, the two methods choose the same locations in this case and thus the lifetime, and also the FIM quality, are the same. From Fig. 17 and 18, we can clearly see that when the number of sensors is 6 or 8 , the system lifetime by $p$-SPEM almost doubles to that of EFI, with a small sacrifice of the FIM quality (5\%). Also when the number of sensors is $10, p$-SPEM achieves a $10 \%$ gain on system lifetime with a $1 \%$ reduction on the FIM quality.

Our experiment completes the cycle in Fig. 3, and calibrates the FEM of the GNTVT with the collected real data. After this field validation, a performance comparison is given in Fig. 19, which compares the 8 deployed positions (chosen by the raw un-calibrated FEM used in the previous cycle) with the 8 positions computed by $p$-SPEM (with the new adjusted FEM). Both the FIM quality and the system lifetime are improved.

In summary, through the field validation on GNTVT, we successfully achieve the mode shapes of GNTVT. Moreover, with the extracted real mode shapes, we not only can evaluate the performance of current sensor placement (8 deployed sensor sets), but also well suggest future adjustment of the placement locations. We would like to admit that by no means we guarantee that in the next round of in-construction monitoring, we will choose the locations suggested by $p$ SPEM. The monitoring system of GNTVT is not a testbed and our choice may still depend a combined consideration of EFI and practical factors such as deployment feasibility, etc. We, however, do provide a concrete option that civil engineering can seriously consider (with a marginal reduction of the FIM quality) with the voice of computer science. Our experience shows our collaborators are happy to have such option.

\section{CONCLUSION}

In this paper, we studied the sensor placement problem for structure health monitoring system not only on computer science efficiency, but also focused on civil requirement. We presented and redeveloped the general framework of the SHM system. We release SPEM which can provide valuable placement quality information in a step by step manner. We showed how topology control, data routing and energy efficiency can be integrated with the SHM framework. We evaluated our scheme with data from an existing SHM system on the Ting Kau Bridge, where we achieved similar placement quality with a 5-fold improvement in energy efficiency. We conducted an experiment with a complete monitoring cycle on the in-built Guangzhou New TV Tower; and the results on the sensor placement have validated the effectiveness of our scheme.

Our efforts have clearly shown that for the sensor network designs, a cross-disciplined knowledge is possible and valuable. Clearly, our algorithms have room for improvement, which will be our direction in the future.

\section{REFERENCES}

[1] Structural Health Monitoring for Guangzhou New TV Tower using Sensor Networks, [online]: http://www.cse.polyu.edu.hk/benchmark/

[2] J. Chang and L. Tassiulas, "Energy Conserving Routing in Wireless ad hoc Networks," in IEEE INFOCOM, 2000. 


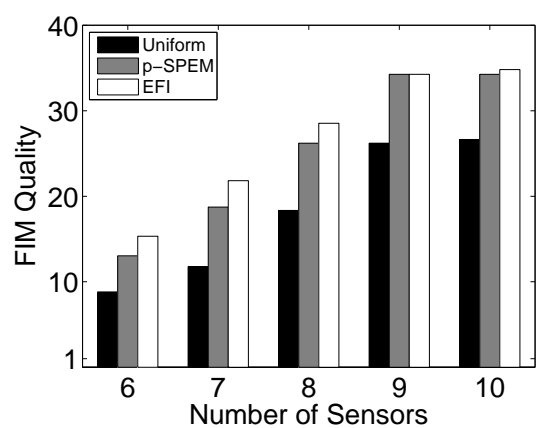

Fig. 17. FIM Quality of different sensor amount.

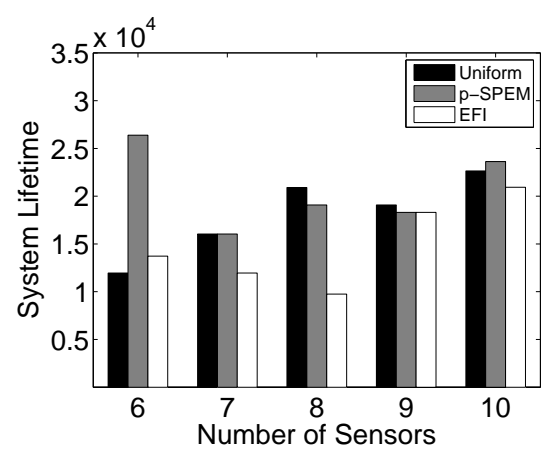

Fig. 18. System lifetime of different sensor amount.

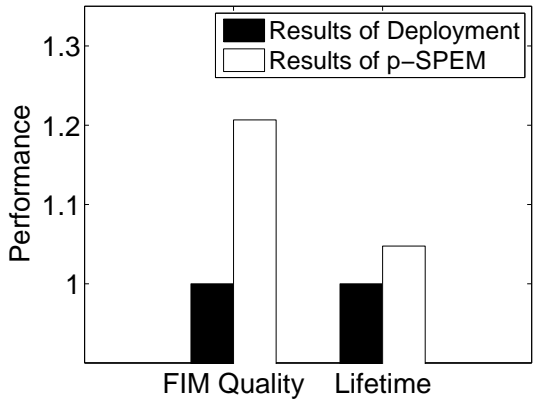

Fig. 19. Eight sensor performance comparison.
[3] K. Chintalapudi, J. Paek, O. Gnawali, T. Fu, K. Dantu, J. Caffrey, R. Govindan, E. Johnson, and S. Masri, "Structural Damage Detection and Localization using NETSHM," in ACM/IEEE IPSN, 2006.

[4] A. Caruso, F. Paparella, L. Vieira, M. Erol, and M. Gerla, "The Meandering Current Mobility Model and Its Impact on Underwater Mobile Sensor Networks," in IEEE INFOCOM, 2008.

[5] G. Hackmann, F. Sun, N. Castaneda, C. Lu, and S. Dyke, "A Holistic Approach to Decentralized Structural Damage Localization Using Wireless Sensor Networks," in IEEE RTSS, 2008.

[6] C. Intanagonwiwat, R. Govindan, and D. Estrin, "Directed diffusion: a scalable and robust communication paradigm for sensor networks," in ACM MobiCom, 2000.

[7] C. Intanagonwiwat, D. Estrin, R. Govindan, and J. Heidemann, "Impact of Network Density on Data Aggregation in Wireless Sensor Networks," in IEEE ICDCS, 2002.

[8] D.C. Kammer, "Sensor Placement for On-orbit Modal Identification and Correlation of Large Space Structures," Journal of Guidance, Control Dynamics, vol.14, pp.251-259, 1991.

[9] S. Kim, S. Pakzad, D. Culler, J. Demmel, G. Fenves, S. Glaser, and M.Turon, "Health Monitoring of Civil Infrastructures Using Wireless Sensor Networks," in ACM/IEEE IPSN, 2007.

[10] A. Krause, C. Guestrin, A. Gupta, and J. Kleinberg, "Near-optimal sensor placements: Maximizing Information While Minimizing Communication Cost," in ACM/IEEE IPSN, 2006.

[11] B. Li, D. Wang, and Y.Q. Ni, "Sensor Placement using EFI Model (SPEM)," [online]: http://www4.comp.polyu.edu.hk/ csdwang/

[12] B. Li, D. Wang, and Y.Q. Ni, "Demo: On the High Quality Sensor Placement for Structural Health Monitoring," in IEEE INFOCOM, 2009.

[13] J. Luo, D. Wang, and Q. Zhang, "Double Mobility: Coverage of the Sea Surface with Mobile Sensor Networks," in IEEE INFOCOM, 2009.

[14] A. Mainwaring, J. Polastre, R. Szewczyk, D. Culler, and J. Anderson, "Wireless Sensor Networks for Habitat Monitoring," in ACM WSNA, 2002.

[15] Y.Q. Ni, J.Y. Wang, and J.M. Ko, "Modal Interaction in Cable-Stayed Ting Kau Bridge," in Proc. the International Conference on Advances in Structural Dynamics, Hong Kong, China, Dec. 2000.

[16] Y.Q. Ni, H.F. Zhou, K.C. Chan, and J.M. Ko, "Modal Flexibility Analysis of Cable-Stayed Ting Kau Bridge for Damage Identification," Computer-Aided Civil and Infrastructure Eng., vol. 23, no. 3, pp. 223236, 2008.

[17] Y.Q. Ni, Y. Xia, W.Y. Liao, and J.M. Ko "Technology Innovation in Developing the Structural Health Monitoring System for Guangzhou New TV Tower,' Structural Control and Health Monitoring, vol. 16, no. 1, pp. 73-98, 2008.

[18] S. Olariu and I. Stojmenovic, "Design Guidelines for Maximizing Lifetime and Avoiding Energy Holes in Sensor Networks with Uniform Distribution and Uniform Reporting," in IEEE INFOCOM, 2006.

[19] S. Singh, M. Woo, and C. Raghavendra "Power-Aware Routing in Mobile Ad Hoc Networks," in ACM MobiCom, 1998.

[20] A. Sankar and Z. Liu, "Maximizing Lifetime Routing in Wireless Ad Hoc Neworks," in IEEE INFOCOM, 2004.

[21] B. Spencer, M. Ruiz-Sandoval, and N. Kurata, "Smart Sensing Technology: Opportunities and Challenges," in Structural Control and Health Monitoring, vol. 11, no.4, pp. 349-368, 2004.

[22] W. Song, R. LaHusen, R. Huang, A. Ma, M. Xu, and B. Shirazi, "Airdropped Sensor Network for Real-time High-fidelity Volcano Monitoring," in ACM MobiSys, 2009.
[23] K. Xu, H. Hassanein, and G. Takahara, "Relay Node Deployment Strategies in Heterogeneous Wireless Sensor Networks: Multiple-Hop Communication Case," in IEEE SECON, 2005.

[24] N. Xu, S. Rangwala, K. Chintalapudi, D. Ganesan, A. Broad, R. Govindan, and D. Estrin, "A Wireless Sensor Network For structural Monitoring," in ACM SenSys, 2004.

\section{APPENDIX}

Each type of mechanical structure has a specific pattern of vibration at a specific frequency. A specific vibration pattern is called a mode shape. Mathematically, mode shapes form a mode information matrix:

$\Phi=\left[\Phi^{1}, \Phi^{2}, \ldots, \Phi^{K}\right]=\left[\begin{array}{cccc}a_{11} & a_{12} & \ldots & a_{1 K} \\ \vdots & \vdots & \vdots & \vdots \\ a_{M 1} & a_{M 2} & \ldots & a_{M K}\end{array}\right]$

in which a column for example $\Phi^{i}=\left[a_{1 i}, a_{2 i}, \ldots, a_{M i}\right]^{\prime}$ represents the $i$ th order mode shape; and a row for example $\left[a_{j 1}, a_{j 2}, \ldots, a_{j N}\right]$ stands for mode shape measurement result from the $j$ th sensor.

Functional output of SHM systems can be represented as:

$$
u=\Phi \cdot q+v
$$

where $u$ is the practical response from the sensors (acceleration etc.), $q$ is the modal response and $v$ is the noise effect.

Linear or spatial independent target modal partition requires that at any time $t$, an estimator $\hat{q}$ can be computed by solving Eq.(1). Assume the sensor noise $v$ to be stationary additive random with zero mean and positive definite covariance intensity matrix $R$. For an efficient unbiased estimator, the covariance matrix of the estimator error is given by:

$$
P=E\left[(q-\hat{q})(q-\hat{q})^{T}\right]=\left[\Phi^{T} \cdot R^{-1} \cdot \Phi\right]^{-1}=Q^{-1}
$$

Here $Q$ is the FIM.

One sensor placement criterion is that the strength of the signals acquired associated with modal characteristics should be as high as possible to reduce the noise effect. Maximizing the FIM determinant does this and can lead to maximizing the spatial independence of the target modal partitions [8];

To compute the set of locations with high FIM, a well accepted scheme is the EFI method. This is an iterative algorithm. In each iteration, the location with the lowest FIM determinant will be removed. The FIM of all the remaining locations will be re-evaluated. Note that after the re-evaluation, the FIM of each location may be substantially changed. For more details on FIM, please refer to [8]. 